\title{
The effect of properties of human body on statistical characteristics channel on-body communication
}

\author{
Esraa H.Kadum ${ }^{1}$ and Haider M. AlSabbagh ${ }^{2}$ \\ 1esraahabeeb63@yahoo.com;'haidermaw@ieee.org, \\ ${ }^{1,2}$ Department of Electrical Engineering, College of Engineering, University of Basra, \\ Basra, Iraq
}

\begin{abstract}
In wireless body area networks, on-body radio propagation channels are typically timevarying, because of the frequent body movements, the one case of human body state is statically case when the body is stand up with its hand along the body, the model taking into account the places of nodes as its shown in this paper, the channel characteristics for statistical one case which may birthing can effect on the design , four channel model line of sight and one sensor (antenna ) put on back which make the channel as non - light of sight .the destine between transmitter and receiver make a very important rule because the path loss dependent on it directly, the system design is many transmitter and one receiver (Master and Slave ) node, single input multi output channel design (SIMO).
\end{abstract}

Keywords: on-body communication, channel model, statistical channel, path loss and power delay profile.

\section{INTRODUCTION}

For a Body Area Network (BAN) channel model, it is required to determine the electromagnetic field at each point on the body, for a given position of the transmitter on the body, this is a big problem numerically, Therefore, it is desirable to drive an analytical expression which performs this objective resolving this problem means solving the Maxwell's equations for each point of the body [1]. Wireless Body Area Networks (WBAN) consist of a number of units placed inside or in proximity of the human body (such as in everyday clothing), and are a natural progression of the WPAN concept [2]. Also known as IEEE 802.15.6, WBAN is a low-frequency technology intended to endow a future generation of shortrange electronics for exchanging information [3] [4]. This paper takes in hand the study one of the body communication (on-body), the channel characterization in the statistical case of human body, this is will do for standing where two hands along the body, the Characterization of the Radio Channel in On-Body Communication[5]. 
The study of statistical channel characteristics like power delay profile, RMS delay spreads, coherence bandwidth and time coherence are performed. Many UWB components and systems are already in the testing and demonstration phases, with actual release dates for final consumer products expected in early 2005. Intel Corporation is working with the industry to enable this exciting technology and help ensure its success. On-body area ultra wideband (UWB) communication is of high importance for promising new biomedical applications $[6,7]$.

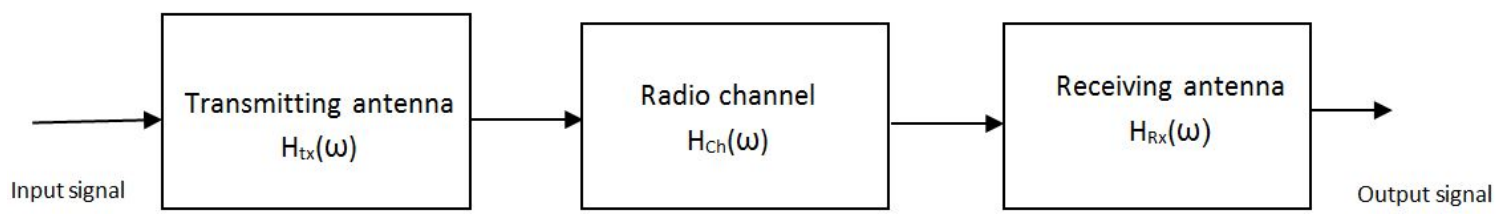

Fig (1) Block diagram representing the radio channel

By taking a single-input, single-output system Fig (1), in which an input signal $x(t)$ is introduced, causing an output signal $y(t)$ to come out. Common assumptions for the system are to be linear and time-invariant, but the ability to release these constraints in the following. Inside the system, some noise could be generated, and added to the "deterministic" part of the output signal. Usually this noise is assumed to be white Gaussian noise, completely uncorrelated with the input signal [8].

A convenient characterization of multi-path propagation, In this model, the time axis is divided into small time intervals called "bins". The received power is integrated within each bin to obtain the energy received as function of the excess delay $[9,10]$. The statistics of the Saleh-Valenzuela model and using the measured Bicone NLOS channel parameters for different case. Specifically the examine of the cumulative distribution function CDF of the mean excess delay, RMS delay spread and the number of paths for the artificial channel [11].

The power delay profile gives information about the delay which a transmitted signal experiences in multipath internment, the PDP is often empirically mo deled by an exponentially decaying [12]. The calculation of the PDP starts from a ray optical point of view. In fig (2), if the positions $R_{x}$ of the receiver and $T_{x}$ of the transmitter are given, the locations of the radiating virtual sources can be calculated using the method of images each virtual source is supposed to transmit at zero delay a Dirac pulse at a carrier frequency $f_{c}$; the pulse needs a delay time until it reaches the receiver [13]. 


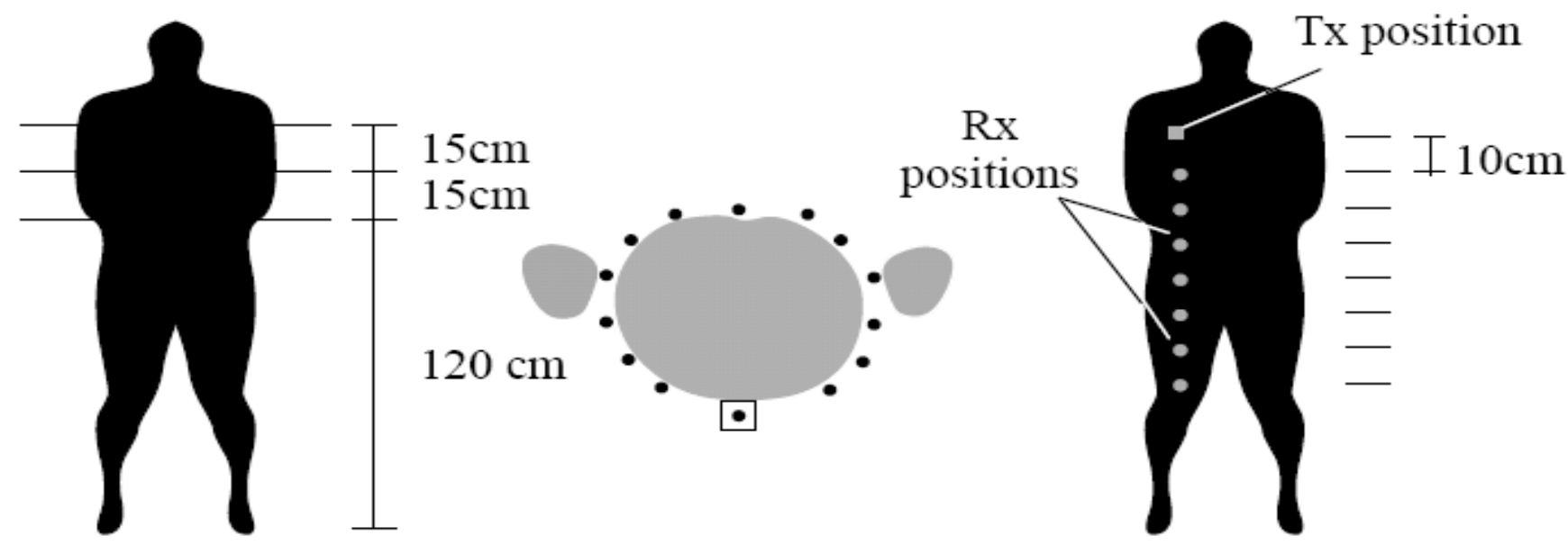

Fig (2) path loss model for standing case

For .. $R_{x}=$ directional antenna,$T_{x}=$ Omni-directional antenna, the fig ( 3 ) shows the principle work of line of sight channel model on body taking account the other obstacles [14].

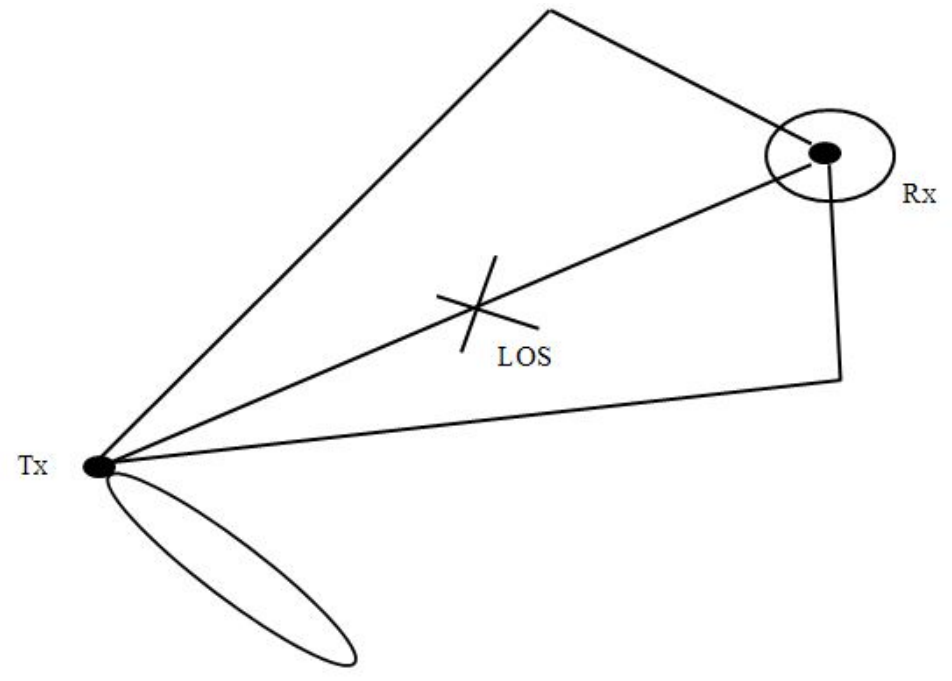

Fig (3) The principle line of sight on body channel (LOS)

\section{EXPERIMENTATION}

\subsection{Multipath Channel Model}

In wireless communications, the multipath propagation phenomenon is when the transmitted signal travels along many different paths to reach the receiver. The presence of multiple paths between transmitter and receiver introduces complexity in the channel modeling [15]. The complexity depends on the distribution of the multipath intensity, relative propagation time of the waves and the bandwidth of transmitted signal. Therefore the time varying properties of the channel must be taken into account in the channel model. The 
presented of a body area UWB multipath channel model based on the classical Saleh Valenzuela model[16] [17] .

The impulse response model is a convenient model to characterize the multipath channel and any deterministic impulse response can be represented by discrete tapped delay line model as long as the system is band limited.

The Saleh-Valenzuela model can be represented as:

$$
h(t)_{i}=X \sum_{l=0}^{L-1} \sum_{k=0}^{k-1} \alpha_{K, 1}^{i} \delta\left(t-T_{1}^{i}-\tau_{k, 1}^{i}\right)
$$

Where $\alpha_{k, 1}^{i}$ represents the multipath gain coefficients, $T_{k, l}^{i}$ represents the delay of the $l^{\text {th }}$ path,$\tau_{k, l}^{i}$ represents the delay $k^{\text {th }}$ multipath component relative to the $l^{\text {th }}$ path arrival time $\left(T_{1}^{i}\right) . X_{i}$ represents the log normal shadowing, and (i) refers to the $i^{\text {th }}$ realization.

For the S-V Channel Model the arrival time and the ray arrival time are Poisson processes:

$$
\begin{gathered}
p\left(\frac{T_{l}}{T_{l-1}}\right)=\Lambda \exp \left[-\Lambda\left(T_{l}-T_{l-1}\right)\right] \ldots \ldots \ldots \mid>0 \\
p\left(\tau_{k, l} / \tau_{(k-1), l}\right)=\lambda \exp \left[-\lambda\left(\tau_{k, l}-\tau_{(k-1), l}\right)\right] \ldots \ldots \mathrm{k}>0
\end{gathered}
$$

So, the design is shown in fig. below:

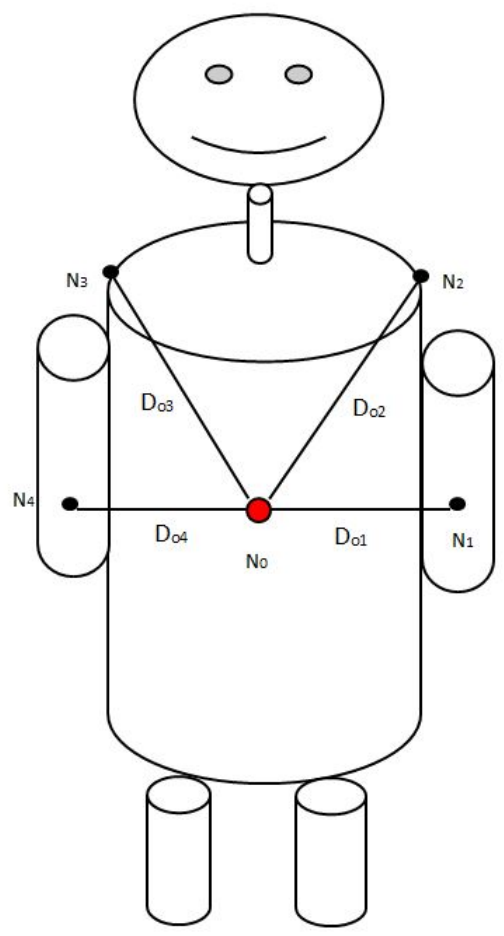

Fig(4) Human body with positions of nodes (antennas) and the desistance between sensors 
For different channel design which are : 4 channel are line- of- sight ( LOS) for distance between transmitter and receiver (node) equal to $D_{1}, D_{2}, D_{3}, D_{4}$.and there is one node which is Non - line - of sight (NLOS) .

CM1: Line Of Sight (LOS) model for $0-30 \mathrm{~cm}$

CM2 : Line Of Sight (LOS) model for $0-30 \mathrm{~cm}$

CM3 : Line Of Sight (LOS) model for $0-70 \mathrm{~cm}$

CM4 : Line Of Sight ( LOS) model for $0-70 \mathrm{~cm}$

CM5 : Non Line of sight (NLOS) for $0-140 \mathrm{~cm}$

For model with parameters $\theta=\left\{\theta_{1}, \theta_{2}, \ldots . . \theta_{p}\right\}$ :

D: the distance between every node and the master node $\left(N_{0}\right), p=$ No. of parameters which are used for optimization and the impulse response of the system.

In practice, the output signal can be written as the sum of the generated noise and a deterministic function of the input signal:

$$
y(t)=n(t)+F[x(t)]
$$

If the system is linear and time-invariant, the function $F$ assumes the form of the convolution between the input signal and the system's impulse response $h(t)$ :

$$
y(t)=n(t)+x(t) \otimes h(t)
$$

By using the software MATLAB the impulse response for four different modified S-V models as shown in fig () it is apparent that the paths in CM1 and CM2 tend to concentrate in two or three paths with smaller delay, whereas in $\mathrm{CM} 3, \mathrm{CM} 4$ have bigger delay because of the more distance. 

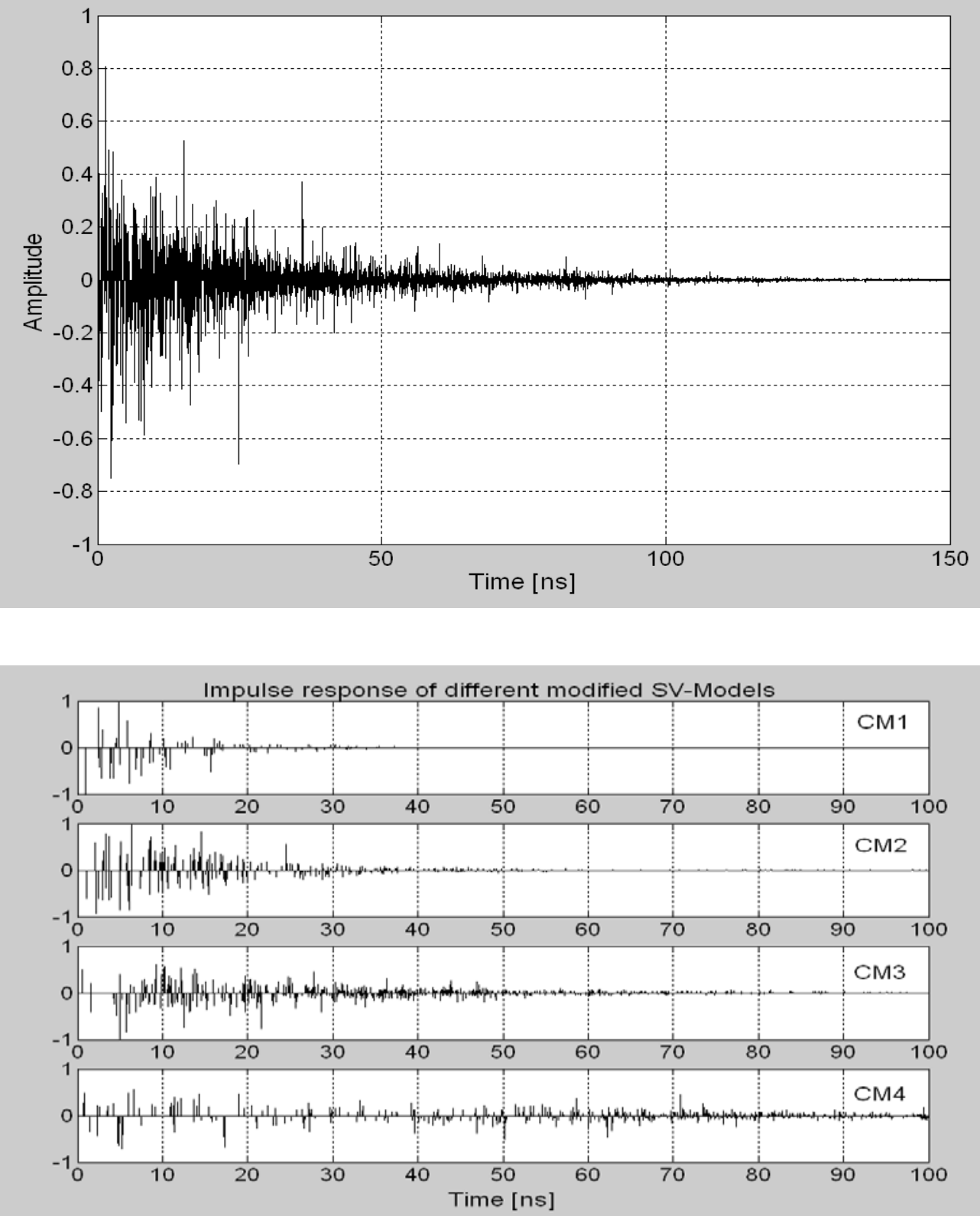

Fig (5) Delay for Saleh -Valenzuela channel model on-body communication CM1-4

For communication between two sensors on the human body, transmitted signals can arrive at the receiver in 3 ways:

- propagation through the body

- diffraction around the body

- Reflections off of nearby scatterers and then back toward the body.

\subsection{Path loss model}

This model represents the exponential decay with distance (expected with diffraction around a cylindrical body), followed by a flat saturation point due to energy received from multipath reflections off nearby scatterers. 


$$
P_{d B}(d)=-10 \log _{10}\left(P_{0} e^{-m_{0} d}+P_{1}\right)+\sigma_{P} n_{P}
$$

The parameters are explained as:

- $\mathrm{P}_{0}$ is the average loss close to the antenna. It will depend on the type of antenna.

- $\mathrm{M}_{0}$ represents the average decay rate in $\mathrm{dB} / \mathrm{cm}$ for the surface wave travelling around the perimeter of the body.

- $\mathrm{P}_{1}$ is the average attenuation of components in an indoor environment radiated away from the body and reflected back towards the receiving antenna.

- $\sigma_{\mathrm{p}}$ is the log-normal variance in $\mathrm{dB}$ around the average representing the variations measured at different body and room locations. This parameter will depend on variations in the body curvature, tissue properties and antenna radiation properties at different body locations.

Wireless dynamic channels can be modeled as linear time- varying systems:

$$
y(t)=\sum a_{i}(t) x\left(t-\tau_{i}(t)\right)
$$

where $a_{i}(t)$ and $\tau_{i}(t)$ are the gain and delay of path $i$.

The time-varying impulse response:

$$
h(t, \tau)=\sum a_{i}(t) \delta\left(\tau-\tau_{i}(t)\right)
$$

So wireless channels are characterized by severe fluctuations in the receive power, i.e., in the strength of the electromagnetic field at the receiver position. The receive power is usually modeled as a combination of three phenomena: path loss, large-scale fading, and small-scale fading.

The path loss describes the distance-dependent power decay of electromagnetic waves.

Let us model the attenuation factor as $d$, where $d$ is the distance of the wave has traveled and denotes the path loss exponent, which is typically assumed to lie between 2 and 4 . The path loss in decibels is then obtained as:

$$
P L_{d B}(d)=P L_{0, d B}+10 n \log _{10} \frac{d}{d_{0}}
$$

In this equation, the path loss exponent $(n)$ is taking as 3.8 where the path loss reference distance $\left(d_{0}\right)$ is $0.1 \mathrm{~m}$ and finally the value the path loss in reference $P L_{0, d B}=43$.

Path loss is the attenuation of an electromagnetic wave as it propagates through space, depending on the environment in which the radio channel is contained, different propagation models are used and consequent path loss will be obtained in a different way. These 
models are mainly empirical with equations based in measurements within a certain frequency band. For urban environments, for example, several models can be used depending on the situation, such as Okumura-Hata Model. Some path loss measurements have already been conducted for on-body elements and some conclusions were taken. As quoted in, some studies have been developed at $2.45 \mathrm{GHz}$ with the body in different positions, propagation paths can be with LOS or NLOS, in other words, transmitter and receiver can "see" each other or do not, respectively.

\section{CONSOLATION}

The distance between antennas (sensors) which is part of channel model and the principles of channel which are line of sight or non-line of sight, this is have direct effect on path loss either the channel was in free space or on body, for channel (1) and channel (2) the delay is smaller than in channel (3) and (4), the effect of human body make the path loss of statistical channel more less than when the channel in free space. The putting of the antennas on the two shoulder and on the two hands make the model more sufficient for this case (standing), when we change the places( legs, knee, waist,....) the model all change and also its statistical characteristics.

\section{REFERENCES}

[1]. A. D. Droitcour, O. Boric-Lubecke, V. M. Lubecke, J. Lin, and G. T. A. Kovacs, "Range correlation and $\mathrm{I} / \mathrm{Q}$ performance benefits in single-chip silicon Doppler radars for noncontact cardiopulmonary monitoring," IEEE Trans. Microw. Theory Tech., vol. 52, no. 3, pp 838-848, Mar. 2004.

[2]. A. A. Serra, P. Nepa, and G. Manara, "On-body antenna input-impedance phase-modulation induced by breathing and heart activity," in Proc. URSI, Jul. 5-11, 2008.

[3]. R. F. Dubrovka and I. B. Shirokov, "On-body antenna for the miners cardiac rhythm sensor," in Proc. Antennas Propag. Conf., Loughborough, 2009, pp. 581-584.

[4]. P. Salonen, Y. Rahmat-Samii, H. Hurme, and M. Kivikoski, "Dual-Band Wearable Textile Antenna", 2004 IEEE AP-S Int. Symp. Dig., volume 1, pp. 463-466,2004.

[5]. N. Jin, and Y. Rahmat-Samii, "Parallel particle swarm optimization and finite-difference time-domain (PSO/ FDTD) algorithm for multiband and wide-band patch antenna designs" IEEE Trans. Antennas Propagat., volume 53, No. 11, pp. 3459-3468, (2005).

[6]. Cheng Tao, Li Jin. Analysis and simulation of RFID anti-collision algorithms .IEEE Proceeding Advanced system. Communi-cation Technology. Phoenix Park,Korea. 2007,pp:697-701 .

[7]. H. Sawada, T. Aoyagi, J. Takada, K. Y. Yazdandoost, and R. Kohno, "Channel model for wireless body area network," in Proc. 2nd Intl. Symp. on Med. Info. and Comm. Tech. (ISMICT), Oulu, Finland, Dec.2007.PP 243-248. 
[8]. T. Zasowski, G. Meyer, F. Althaus, and A. Wittneben, "Propagation effects in uwb body area networks," in IEEE Intl. conf. on Ultra -wideband, Sept. 2005.

[9]. A. Fort, J. Ryckaert, C. Desset, P. D. Doncker, P. Wambacq, and L. V. Biesen, "Ultra-wideband channel model for communication around the human body," IEEE journal on selected areas in comm., vol. 24, pp. 927-933, 2006.

[10]. B. Zhen, M. Kim, J. Takada, and R. Kohno, "Characterization and modeling of dynamic onbody propagation at $4.5 \mathrm{GHz}$," IEEE Antennas Wireless Propagat. Lett., vol. 8, pp. 1263-1267, 2009.

[11]. J. Hagedorn, J. Terrill, W. Tang, K. Sayrafian, K. Y. Yazdandoost, and R. Kohno, "A statistical path loss model for MICS," IEEE 802.15-08-0519-01-0006, Sept. 2008.

[12]. S. L. Cotton and W. G. Scanlon, "Characterization of the on-body channel in an outdoor environment at $2.45 \mathrm{ghz}$, " in European conf. on antennas propagation, 2009, pp. 722-725.

[13]. M. Kim and J. Takada, "Statistical model for $4.5 \mathrm{GHz}$ narrowband on- body propagaton channel with specific actions," IEEE Antennas Wireless Propagat. Lett., vol. 8, pp. 1250-1254, 2009.

[14]. A. Fort, J. Ryckaert, C. Desset, P. D. Doncker, P. Wambacq, and L. V. Biesen, "Ultra-wideband channel model for communication around the human body," IEEE journal on selected areas in comm., vol. 24, pp. 927-933, 2006.

[15]. R. Istepanian, E. Jovano V, Y. Zhang, "Guest Editorial Introduction to the Special Section on M-Health: Beyond Seamless Mobility and Global Wireless Health-Care Connectivity " in IEEE Transactions on Informati on Technology in Biomedicine, 8(4): 405 - 414, Dec . 2004.

[16]. D. Raskovic, T. Martin, E . Jovanov, "Medical Monitoring Applications for Wearable Computing," in The Computer Journal, July 2004, 47(4):pp. 495-504.

[17]. Mehmet. Yuce, Ho. Keon g, and Moo. Chae, "Wideband Communication for Implantable and Wearable Systems", in IEEE TRANSACTIONS ON MI CROWAVE THEORY AND TECHNIQUES, VOL. 7 , NO10, OCTOBER 2009. 